\title{
Use of Image Analysis to Understand Enzyme Stability in an Aerated Stirred Reactor
}

Dias Gomes, Mafalda; Moiseyenko, Rayisa P.; Baum, Andreas; Jørgensen, Thomas M.; Woodley, John M.

\section{Published in:}

Biotechnology Progress

Link to article, DOI:

10.1002/btpr.2878

Publication date:

2019

Document Version

Peer reviewed version

Link back to DTU Orbit

Citation (APA):

Dias Gomes, M., Moiseyenko, R. P., Baum, A., Jørgensen, T. M., \& Woodley, J. M. (2019). Use of Image Analysis to Understand Enzyme Stability in an Aerated Stirred Reactor. Biotechnology Progress, 35(6), [e2878]. https://doi.org/10.1002/btpr.2878

\section{General rights}

Copyright and moral rights for the publications made accessible in the public portal are retained by the authors and/or other copyright owners and it is a condition of accessing publications that users recognise and abide by the legal requirements associated with these rights.

- Users may download and print one copy of any publication from the public portal for the purpose of private study or research.

- You may not further distribute the material or use it for any profit-making activity or commercial gain

- You may freely distribute the URL identifying the publication in the public portal 


\title{
Use of Image Analysis to Understand Enzyme Stability in an Aerated Stirred Reactor
}

\author{
Mafalda Dias Gomes ${ }^{1}$, Rayisa P. Moiseyenko², Andreas Baum ${ }^{2}$, Thomas M. Jørgensen ${ }^{2}$, and John M. Woodley ${ }^{1 *}$ \\ ${ }^{1}$ Department of Chemical and Biochemical Engineering, Technical University of Denmark, Building 229, Søltofts Plads, DK-2800 \\ Kgs. Lyngby, Denmark \\ ${ }^{2}$ Statistics and Data Analysis, Department of Applied Mathematics and Computer Science, Technical University of Denmark, Richard \\ Petersens Plads, 2800, Kgs. Lyngby, Denmark \\ * Corresponding author: jw@kt.dtu.dk
}

\section{Abstract}

Efficient regeneration of $\mathrm{NAD}(\mathrm{P})^{+}$cofactors is essential for large-scale application of alcohol dehydrogenases due to the high cost and chemical instability of these cofactors. NAD $(P)^{+}$can be regenerated effectively using NAD(P)H oxidases that require molecular oxygen as a co-substrate. In large-scale biocatalytic processes, agitation and aeration are needed for sufficient oxygen transfer into the liquid phase, both of which have been shown to significantly increase the rate of enzyme deactivation. As such, the aim of this study was to identify the existence of a correlation between enzyme stability and gas-liquid interfacial area inside the bioreactor. This was done by measuring gas-liquid interfacial areas inside an aerated stirred reactor, using an in situ optical probe, and simultaneously measuring the kinetic stability of $\mathrm{NAD}(\mathrm{P}) \mathrm{H}$ oxidase. Following enzyme incubation at various power inputs and gas-phase compositions, the residual activity was assessed and video samples were analysed through an image processing algorithm. Enzyme deactivation was found to be proportional to an increase in interfacial area up to a certain limit, where power input appears to have a higher impact. Furthermore, the presence of oxygen increased enzyme deactivation rates at low interfacial areas. The areas were validated with defined glass beads and found to be in the range of those in large-scale bioreactors. Finally, a correlation between the enzyme half-life and specific interfacial area was obtained. Therefore, we conclude that the method developed in this contribution can help to predict the behaviour of biocatalyst stability under industrially relevant conditions, concerning specific gas-liquid interfacial areas. 


\section{Introduction and Motivation}

Oxidoreductases (EC 1) are enzymes that catalyse oxidation and reduction reactions. As biocatalysts, these enzymes have attracted significant interest for industrial application in the synthesis of chemicals not only for pharmaceuticals but also for the production of lower priced chemicals ${ }^{1-6}$. Since these enzymes are cofactor-dependent and the particular cofactors involved have a high cost and are required in stoichiometric amounts, there is a need to regenerate them ${ }^{7}$. Therefore, cofactor regeneration systems are essential to lower the cost of such processes and drive reactions to completion by shifting the equilibrium towards the product of interest ${ }^{8}$.

Alcohol dehydrogenases (ADHs) are oxidoreductases enzymes (EC 1.1.1.1) that can catalyse the oxidation of alcohols to ketones (or aldehydes), which are important building blocks for the production of many important chemicals ${ }^{9,10}$. The $\mathrm{NAD}(\mathrm{P})^{+}$cofactor is necessary as an electron acceptor (Figure 1). As mentioned previously, a cofactor regeneration system is required for practical application of these enzymes and there are several regeneration strategies that can be used ${ }^{10-12}$. One of particular interest is the use of $\mathrm{NAD}(\mathrm{P}) \mathrm{H}$ oxidases (NOXs) ${ }^{13,14}$. NOXs have the great benefit of using molecular oxygen as an electron acceptor, which is inexpensive and abundant. Additionally, it favours the oxidative direction of the $\mathrm{ADH}$ reaction since the cofactor system is thermodynamically favourable driven molecular oxygen $(\text { Figure 1) })^{12,15,16}$.

When NOX is used for cofactor regeneration in industrially relevant $\mathrm{ADH}$ catalysed oxidations, oxygen needs to be supplied at high rates in order to achieve the target productivities ${ }^{17-19}$. For example, for a medium value product, a productivity of $10 \mathrm{~g} \mathrm{~L}^{-1} \mathrm{~h}^{-1}$ is required thus, to produce a molecule with a molecular weight of $100 \mathrm{~g} \mathrm{~mol}^{-1}$, an oxygen transfer rate of $100 \mathrm{mmol} \mathrm{L}^{-1} \mathrm{~h}^{-1}$ is necessary (for a stoichiometry of $1 \mathrm{~mol}$ of oxygen per mol of product). However, dissolved levels of oxygen in solution are insufficient to achieve this. As a result, aeration and stirring are essential both to achieve sufficient oxygen transfer rates and also to ensure a constant supply of oxygen ${ }^{20}$. Generally, oxygen is supplied by sparging air bubbles into the liquid and stirring is used to break the bubbles, increase dispersion and consequently creating a high gas-liquid interfacial area. In this environment, the biocatalyst is inevitably exposed to gas-liquid interface. Some enzymes are known to deactivate in the presence of such interfaces ${ }^{21-26}$. However, the correlation between protein stability and gas-liquid interfacial area is not well understood. It should be noted that a protein in solution exposed to gas bubbles in a turbulent flow inside a bioreactor is very different from a quiescent liquid enzyme solution exposed to a gas-liquid interface at the surface of the reaction liquid. Therefore, to study the deactivation of an enzyme in a tube with 
an open lid is not sufficient to understand the effect of real process conditions on the biocatalyst. Thus, we argue that enzymes have to be exposed to conditions in the laboratory similar to those at an industrial scale.

The method developed here uses an endoscopic probe connected to a high speed camera to measure the size of bubbles inside the reactor. This technique has been applied previously to study the influence of different process conditions, such as agitation speed and gas flow rate, on bubble size distribution ${ }^{27,28}$. In the present work, we go a step further and an image processing algorithm was used to quantify the interfacial area at different oxygen supply conditions, which was subsequently correlated with the deactivation rate of the biocatalyst. NOX was selected for investigation not only because of its industrial relevance but specifically because it is an oxidase. This is particularly interesting since oxygen is required for the reaction to take place whilst at the same time it can have a negative effect on the enzyme stability. The aim of this contribution is to better understand the correlation of enzyme stability and gas-liquid interfacial area, under industrial process conditions. So the present work investigates the deactivation of a water-forming NOX from Streptococus mutans

${ }^{29}$ at different power inputs (defined as power input per volume (P/V)). Additionally, air and nitrogen were sparged to study the influence of oxygen on the deactivation of the enzyme.

\section{Materials and Methods}

\section{Materials}

A water forming NAD(P)H oxidase (NOX) from Streptococcus mutans ${ }^{29}$ was kindly supplied by InnoSyn (Geleen, The Netherlands) as a cell free extract (CFE) formulation. Potassium dihydrogen phosphate and dipotassium hydrogen phosphate, purchased from VWR (Radnor, PA, USA), were used to prepare potassium phosphate buffer. NADPH tetrasodium salt ( $\beta$-nicotinamide-adenine dinucleotide phosphate, reduced) was acquired from VWR (Radnor, PA, USA). NOX activity was on average $850 \mathrm{U} / \mathrm{mL}_{\mathrm{CFE}}$. One unit corresponds to $1 \mu \mathrm{mol}$ of NADPH consumed per minute.

\section{Bioreactor and Optical Method Setup}

NOX was incubated in a stirred tank reactor, with a total volume of $250 \mathrm{~mL}$, designed and built in-house. The bioreactor contained two Rushton turbines, two baffles and a metal sparger. The optical probe for visualising bubbles inside the reactor (Figure 2) consisted of a high speed camera, an endoscope and a light source. The 
camera was a monochrome Phantom Miro C110 (Vision Research, Wayne, NJ, USA) capable of capturing videos of 800 fps (frames per second) at a resolution of 1289 x 1024 pixels. It was connected to a computer through an Ethernet cable to collect and store the video data. It was attached to the endoscope by a C-mount lens adaptor. The endoscope was a 10 mm high definition (HD) laparoscope from Olympus (Tokyo, Japan). It was positioned on the side of the first impeller so that the bubbles were dragged horizontally in that region, avoiding attachment to the probe and blocking the optics. In order to illuminate the bioreactor and create a contrast between the gas bubbles and the liquid, a back lighting source (LED matrix from Cree, Durham, NC, USA) was installed inside the vessel. The LED was placed in a custom made stainless steel housing. It could supply 24 Watts and 2200 lumens and was connected to an external power supply that enabled control of the light intensity under different incubation conditions.

\section{Stirring and Sparging Conditions}

In order to study the effect of stirring and sparging on the stability of NOX, the power input to the reactor and the composition of the gas phase were varied. Experiments were carried out at power inputs of $0.5,1,1.5,2,3$ and $4 \mathrm{~W} \mathrm{~L}^{-1}$ and with a constant sparging rate of $1 \mathrm{vvm}$, using both air (21\% oxygen, $79 \%$ nitrogen) and pure nitrogen. The power input per volume values $(P / V)$ were defined based on the range used for industrial bioreactors (average ranges from 1 to $2 \mathrm{WL}^{-1}$ ca. $100 \mathrm{~m}^{3}$ vessels $\left.{ }^{30,31}\right)$ and the impeller stirring speed $(N)$ was estimated from equation (1).

$$
N=\sqrt[3]{\frac{P}{N_{p} \cdot \bar{\rho} \cdot D^{5}}}
$$

$P$ is function of the impeller power number $\left(N_{p}\right)$, average density of the medium $(\bar{\rho})$, impeller rotational speed $(N)$ and impeller diameter $(D) . N_{p}$ depends on the impeller type and its Reynolds number (equation (2)). For the Rushton turbine used, the impeller number was assumed 5 from the graphic presented by Paul et al. (2004) $\left(D=0.025 \mathrm{~m}\right.$ and $\left.\operatorname{Re}>10^{3}\right)$. All experiments were carried out in dilute aqueous solutions and so the density and viscosity of water at $25{ }^{\circ} \mathrm{C}$ were assumed for the calculations.

$$
R e=\frac{\bar{\rho} \cdot N \cdot D^{2}}{\mu}
$$




\section{NOX Incubation Experiments}

For all conditions tested, the enzyme was incubated in the reactor, with a working volume of $150 \mathrm{~mL}$, at a concentration of $1 \mathrm{~g}_{\mathrm{CFE}} \mathrm{L}^{-1}$ in $50 \mathrm{mM}$ phosphate buffer at $\mathrm{pH}$ 7.5. Incubations were performed at $25^{\circ} \mathrm{C}$ for 6 hours. A control experiment was performed under the same conditions but in a quiescent solution (without stirring and sparging). The NOX solution was sampled at regular intervals and stored on ice to stop further enzyme inactivation. Subsequently, the enzyme solution was assayed for residual activity, relative to a sample with no incubation (time zero), by following the NADPH consumption at $340 \mathrm{~nm}$ in a spectrophotometer. Activity was measured at $25{ }^{\circ} \mathrm{C}$ using $1 \mathrm{~mL}$ cuvettes, $50 \mathrm{mM} \mathrm{KPi} \mathrm{pH}$ 7.5, $0.20 \mathrm{mM} \mathrm{NADPH}$ and $0.05 \mathrm{~g}_{\mathrm{CFE}} \mathrm{L}^{-1}$. At the same time the protein solution was sampled to measure its activity, a video sample was taken from the reactor in order to measure the area of the corresponding gas-liquid interface. The videos captured a minimum image resolution of $800 \mathrm{fps}$ and were stored for data analysis. Next, these were analysed using image analysis algorithms to detect the bubbles' diameter and further generate a bubble size distribution for each operational condition. The image analysis technique applied in this work is described in the next section. The gas holdup was determined experimentally by the difference between the liquid level in the reactor, with and without aeration and stirring. Measurements were carried out in triplicate. The data flow diagram in Figure 3 illustrates the data collection and analysis procedure.

\section{Image Analysis for Bubble Size Determination}

The number of visible bubbles in each video frame was estimated using automated image analysis. As it can be seen in Figure 4, the appearance of the bubbles varies depending on their size and distance to the focal plane. In order to observe a bubble, a sufficiently large part of an elliptic arc is required to be present in the image. From experimentation, it was found that having at least 120 degrees of arc identified objects in the image which, by human inspection, were considered to be gas bubbles. For the identified bubbles, the primary axis of the corresponding elliptic fit was used to estimate the size of the corresponding bubble.

The essential part of the image processing analysis was based on an elliptical arc detector, previously reported by Pătrăucean et al. (2012). Furthermore, the algorithm implementation was based on the so-called Helmholtz principle ${ }^{33}$. This principle ensured that the image features used to identify the arc were unlikely to occur randomly (i.e. as a result of noise), which reduces the number of false positive detections. Specifically, the elliptic arc detector operates on spatial gradients calculated from the grey scale images (video frames). In order to ensure robust detection of the bubbles, 
independent of their size and distance to the focal plane, the image analysis was performed at different scales (resolutions) $^{34}$. The amount of upscaling/downscaling needed was determined by comparing the detected bubbles on a representative set of video frames with those identified by visual observation of the images. The selected scaling factor was chosen by sequentially applying factors in the range of 0.1 to 4 , with a step interval of 0.1 . From visual inspection, it was decided that factor 3 gave the best performance. Finally, results showed that it was sufficient to operate at only one scale, therefore the image size was always upscaled by a factor of 3 .

During video recording, inhomogeneous illumination of the sample region caused a background pattern with several circular shaped arcs, visible in the outer regions (Figure 4(a)). In order to suppress these images and improve the contrast, the background patterns were subtracted prior to analysis (Figure 4(b)). Furthermore, the algorithm for identifying arc structures detected, in many cases, a double border of the bubbles that led to double detection/counting. This issue was solved by checking whether a detected elliptic structure contains another elliptic shape in its interior. In such cases, the smaller one was neglected (Figure 4(c)). Finally, when calculating the frequency distribution of bubble sizes, a minimum of 800 frames was used for the analysis of each video.

\section{Calibration and Validation of the Image Analysis Algorithm}

In order to calibrate the estimated bubble sizes obtained from image analysis, measurements using glass beads with known diameters, under the same operational conditions and reactor configuration of the incubation experiments, were performed

27. The glass beads were purchased from VWR (Radnor,PA,USA) and had diameters of 1 and $2 \mathrm{~mm}$. For each glass bead size, the suspensions were placed in a $250 \mathrm{~mL}$ bioreactor with a working volume of $150 \mathrm{~mL}$ of water. A power input of $2 \mathrm{~W} \mathrm{~L}^{-1}$ was used and number, area and volume distributions were obtained. The mode of the peaks from the obtained size distributions represent the average size of the detected beads. For the $1 \mathrm{~mm}$ glass beads, the modes were estimated to be 40.7, 41.3 and 42.0 image pixels for the number, area and volume distributions, respectively. Similarly, for the 2 $\mathrm{mm}$ beads, the mode of the peaks were found to be $84.9,86.8,85.4$ image pixels, for number, area and volume distributions, respectively. From these results, it was estimated that $1 \mathrm{~mm}$ corresponds to 41 pixels, which was consistent for both bead sizes tested.

The total gas volume in the reactor $\left(\mathrm{V}_{\text {gas }}\right)$ at different power inputs was previously estimated from experimentally measured gas holdups (Gas holdup $=\mathrm{V}_{\text {gas }}+\mathrm{V}_{\text {liquid }} / \mathrm{V}_{\text {liquid }}$ ) and a positive linear trend was observed (Figure 5). To validate 
the image processing algorithm, the same volumes were independently calculated using the volume distribution of gas bubbles, obtained through image analysis, and the trends compared. As illustrated in Figure 5, it was observed that, for power inputs up to $3 \mathrm{WL}^{-1}$, the total volume of gas determined by image analysis increases linearly with the power input, in accordance with the experimentally measured data. However, for higher power inputs $\left(\geq 3 \mathrm{WL}^{-1}\right)$, the trend appears to reach a plateau. The likely cause of this deviation is that the number of bubbles captured were so numerous that individual bubbles became indistinguishable in the recorded videos. In other words, the captured images became saturated with gas bubbles, such that the image analysis method could no longer capture the increase of gas volume with an increase in power input. Nevertheless, the image processing algorithm appears to be valid in that it was able to show the same trend as the experimental data. However, due to saturation at high power inputs, image analysis was only applied to predict Sauter mean diameters, which, together with the experimental values of gas holdup, were used to estimate specific interfacial areas.

\section{Bubble Size Distributions}

In order to estimate the Sauter mean diameter, size histograms of the bubbles, detected by the video analysis, were calculated. The observed diameters of the gas bubbles were divided into a series of intervals with the same size termed bins ( $i$ ). The probability density function for the bubbles diameter based on the number of detected bubbles is given by equation (3),

$$
P_{N}\left(\bar{d}_{B_{j}}\right)=\frac{N_{i}}{\sum_{i} N_{i}}
$$

where $\bar{d}_{B_{j}}$ is the average size of a bubble and $N$ is the number of bubbles in bin $i$. By definition, the sum of all probabilities must be equal to one (equation (4)).

$$
\sum_{i} P_{N}\left(\bar{d}_{B_{j}}\right)=1
$$

As it is intended to estimate the gas-liquid surface area, it is important to evaluate the impact of the area and volume distribution on the bubbles' diameter estimation. The probability density functions based on the surface area and volume of the bubbles are given by equations (5) and (6). 


$$
\begin{aligned}
& P_{A}\left(\bar{d}_{B_{j}}\right)=\frac{N_{i} \cdot \bar{d}_{B_{j}}{ }^{2}}{\sum\left(N_{i} \cdot \bar{d}_{B_{j}}{ }^{2}\right)} \\
& P_{V}\left(\bar{d}_{B_{j}}\right)=\frac{N_{i} \cdot \bar{d}_{B_{j}}{ }^{3}}{\sum\left(N_{i} \cdot \bar{d}_{B_{j}}{ }^{3}\right)}
\end{aligned}
$$

\section{Gas-Liquid Interfacial Area Determination}

According to the two-film theory, the transport of oxygen from gas to liquid phase is dependent on the diffusion coefficient through the liquid film, the concentration gradient of oxygen and the interfacial area available ${ }^{35}$. The theory assumes that the gradient on the gas side is insignificant. The oxygen transfer to the liquid can be described by equation (7), where the mass transfer coefficient $\left(k_{L}\right)$ and the specific surface area $(a)$ are treated as one overall volumetric mass transfer coefficient known as the $k_{L} a$.

$$
\text { OTR }=k_{L} a \cdot\left(\left[O_{2}\right]^{*}-\left[O_{2}\right]\right)
$$

In order to obtain high oxygen transfer rates, a high $k_{L} a$ is necessary, which is dependent on the available transfer area per volume of liquid, $a$. In other words, a large gas-liquid interfacial area increases the $k_{L} a$. The present work aims to estimate this interfacial area and, for that, the following definition was used.

$$
a=\frac{A}{V_{l}}=\frac{6 \cdot \varepsilon}{(1-\varepsilon) \cdot d_{32}}
$$

The specific interfacial area $(a)$ is the ratio of the total interfacial area in a gas liquid dispersion $(A)$ and the liquid volume $\left(V_{l}\right)$. It is dependent on the gas holdup $(\varepsilon)$ and on the average bubble diameter that is commonly expressed by the Sauter mean diameter $\left(d_{32}\right)^{36,37}$. The gas holdup is the ratio of the volume of gas to the total volume of the gas-liquid dispersion. The Sauter mean diameter is the mean diameter based on the average of gas-liquid surface area and is presented in equation (9). This can be estimated based on bubble size distributions in the reactor.

$$
d_{32}=\frac{\sum N_{i} d_{B, i}^{3}}{\sum N_{i} d_{B, i}^{2}}
$$

The gas holdup and the average bubble size diameter were experimentally determined in this contribution, as mentioned in the previous section. 


\section{Results and Discussion}

\section{Effect of Aeration and Mixing on NOX Kinetic Stability}

First, by comparing the NOX incubation in quiescent solution with the different mixing conditions, it is clearly observed that aeration and stirring have a negative effect on enzyme stability (Figure 6 (left)). Secondly, results showed that the residual activity of NOX decreases faster with an increasing power input. Furthermore, to investigate if the presence of oxygen had an influence in the NOX deactivation, the feed gas composition was varied between air (21\% of oxygen, 79 $\%$ nitrogen) and pure nitrogen. It was found that NOX was more stable when nitrogen was sparged to the reactor (Table 1). Nevertheless, to better compare the results at power inputs with different feed gases, the deactivation rate constant and enzyme half-life were calculated for all cases. As can be seen in Figure 6 (right), it was observed that the deactivation kinetics were close to first-order with respect to enzyme concentration. Accordingly, first-order deactivation kinetics were assumed and the deactivation rate constant $\left(k_{d}\right)$ was estimated from the slope of the natural logarithm of the residual activity over time ${ }^{38}$. The figures for all investigated conditions were presented in Table 1.

When air was supplied, results from Table 1 revealed that the half-life of NOX decreased with an increase in power input. With respect to nitrogen, the same behaviour was observed for the lowest power inputs tested, although, from 2 to $4 \mathrm{WL}^{-}$

${ }^{1}$, it appears that the half-life slightly increases. Nevertheless, the values obtained are within experimental variability, so a clear conclusion cannot be drawn for higher mixing conditions when nitrogen was supplied. It can also be seen that NOX is generally more stable with nitrogen (compared to oxygen), as mentioned before. However, the difference in the enzyme stability between the two gases tends to decrease with an increase in power input. These results indicate that there are several phenomena affecting protein deactivation and that the presence of oxygen is not the sole cause. As the power input increases, the gas-liquid interfacial area also increases and the enzyme is more exposed to the interface, which enhances its deactivation. In order to further understand the reasons of NOX deactivation, the interfacial area in the reactor was measured and correlated with the enzyme half-life.

\section{Determination of the Gas-Liquid Interfacial Area in the Bioreactor Setup}

When air is sparged to a stirred tank, small gas bubbles are created and the biocatalyst is inevitably exposed to gas-liquid interface. Preliminary tests were carried out to decide whether the aeration or the stirring had a major effect on changing the interfacial area in the setup. It was found that the stirring speed had a higher impact on changes in gas-liquid interfacial 
area and these differences could be detected by the optical probe (Figure 7). Therefore, in the experiments performed, different interfacial area conditions were obtained by changing the stirring speed of the impeller. The stirring speed is reported in terms of power input (power input per volume of reactor $(\mathrm{P} / \mathrm{V})$ ) to compare the results directly with those applied at industrial scale. Thus, the power inputs used in the experiments were selected based on the values used in industrial reactors. However, higher power inputs were also tested to better understand the effect of high interfacial area on protein stability.

From the video samples, bubble size distributions for all power inputs tested were obtained using the offline image analysis technique (Figure 8). The number, area and volume distributions were calculated for all conditions tested. Although air and nitrogen were used, no difference was found in the bubble size between these two gases, as it was expected. Furthermore, this analysis was performed assuming spherical bubbles. This assumption was verified within the mixing conditions tested by the videos collected, as observed for example in Figure 4 and Figure 6 . Thus, the distributions obtained showed the same shape as the ones observed at the same reactor position in the work developed by Laakkonen et al. (2005), which reports bubble size distributions in different zones in an aerated stirred reactor.

With the purpose of estimating the gas-liquid interfacial area for all mixing conditions, the area and volume distributions are particularly important. The distributions take into consideration the large bubbles in the reactor, which contribute more to the total interfacial area. It can be seen from the number distribution that small bubbles dominate the reactor. Whereas looking into the area and volume distributions, the mode increases. Therefore, the Sauter mean diameter $\left(d_{32}\right)$ was used to calculate an average size of the bubbles since it is based on the mean diameter from the area and volume distributions. The results obtained are presented in Figure 8 and show that the $d_{32}$ does not change significantly with an increase in power input. However, more bubbles are detected in the reactor at higher power inputs, as observed in Figure 6. This can be explained by the increase in gas holdup with the increase in power input. At higher stirring speeds, the bubbles were kept longer in the liquid, increasing the interfacial area available. Therefore, the gas-liquid interfacial area is larger at higher power input because the mixing increases the residence time of the bubbles in the reactor, measured by the gas holdup.

Finally, the interfacial area was calculated using the Sauter mean diameter $\left(d_{32}\right)$ and the gas holdup. This was expressed in terms of specific interfacial area, which is the ratio of the gas-liquid interfacial area and to reactor volume. In Figure 
9, a correlation was established and it was found that the specific interfacial area $(a)$ increased with an increase in power input. Indeed, these two parameters cannot be separated when the bioreactor is aerated.

Finally, comparing the results with mechanically agitated contactors, it was found that the specific interfacial areas achieved in this study were within the range of those observed in large scale reactors $\left(20-200 \mathrm{~m}^{-1}\right.$, from Paul et al. $(2004))^{30}$. This result is very promising since it demonstrates that specific interfacial areas of an industrial aerated stirred reactor can be mimicked at a small-scale. The method developed here allows enzyme incubation close to industrially relevant conditions, using less enzyme compared to larger reactors (e.g. pilot scale). Therefore, this gives the possibility of studying protein stability in the presence of gas-liquid interfaces in a turbulent flow regime prior to process scale-up. Based on these findings, we propose a scale-down approach that is illustrated in Figure 10. The proposed scale-down method starts with identification of the oxygen requirements for the oxidation reaction. Next, the oxygen transfer rate must be defined and, based on this, the required specific interfacial area $(a)$ should be estimated. This specific interfacial area must then be mimicked at laboratory scale and the enzyme solution incubated at different areas of gas-liquid interface. From the measured kinetic stability of the enzyme at different specific interfacial areas, a correlation between enzyme half-life and gas-liquid interfacial area can be proposed. This correlation can help to understand the stability of the biocatalyst under industrial process requirements and facilitate the definition of adequate process conditions. Furthermore, the process knowledge obtained can be used to inform protein engineering research towards modifying the enzyme according to industrial needs.

\section{Correlation of NOX Kinetic Stability with Gas-Liquid Interfacial Area}

With the purpose of understanding the deactivation of NOX at gas-liquid interfaces, a correlation between the enzyme half-life and the specific interfacial area is presented in Figure 11. As expected, results show that the NOX half-life decreases with an increase in interfacial area. The same behaviour is observed in the presence of both air and nitrogen. Additionally, two distinct regions can be seen in the plot: a regime above and below $a=75 \mathrm{~m}^{-1}$.

In the regime at low interfacial area $\left(<75 \mathrm{~m}^{-1}\right)$, sparging air decreases the enzyme half-life in comparison with supplying nitrogen. The result indicates that the oxygen present in the feed gas may be causing overoxidation of the cysteine residue present in the enzyme active site ${ }^{40}$. Water-forming NOX enzymes, have a conserved cysteine residue that is catalytically active. During the redox cycle, the cysteine can be overoxidized producing sulfinic and sulfonic acid that deactivates the 
enzyme ${ }^{41}$. On the other hand, at high gas-liquid interfacial area $\left(>75 \mathrm{~m}^{-1}\right)$, the difference in the NOX half-life between the two gases decreases. This observation suggests that the presence of oxygen is not the major reason for NOX deactivation but rather the presence of the gas-liquid interface per se.

Looking more closely into the correlation, for $a>75 \mathrm{~m}^{-1}$, the stability of NOX reaches a plateau, even though the interfacial area increases. Therefore, it would appear that stirring also influences the deactivation of NOX. Indeed, the interfacial area is changed by increasing the stirring speed and in these results the two factors cannot be separated. Thus, in order to further investigate the effect of agitation, an experiment without aeration was run in the same setup at power inputs of $0.5,1$ and $2 \mathrm{WL}^{-1}$.

Results presented in Table 1 show that for the lower power input the presence of air reduces the half-life of NOX, which confirms that oxygen has an effect on NOX stability. However, for 1 and $2 \mathrm{WL}^{-1}$, the presence of a gas phase did not change NOX stability significantly. This behaviour indicates that the presence of gas is not the dominant cause of protein inactivation when the system is being agitated. Therefore, the deactivation of NOX would appear to be caused by three distinct parameters: (1) stirring, (2) presence of a gas-liquid interface and (3) the presence of oxygen. In order to understand to which extent each of these parameters deactivate the enzyme, they would need to be investigated independently. Recent studies on NOX using a bubble column showed that the presence of a gas-liquid interface with no agitation deactivates the enzyme ${ }^{42}$. Nevertheless, the method developed here allows the study of the influence of these parameters together on the biocatalyst stability, which is extremely relevant to evaluate the deactivation of enzymes under real process conditions.

\section{Conclusions}

In conclusion, we have developed and applied an experimental laboratory method to study the kinetic stability of enzymes toward gas-liquid interfaces, which mimics specific gas-liquid interfacial areas of industrial bioreactors. The distinct aspect of this method is that it uses an image processing algorithm for in situ quantification of the gas-liquid interfacial area in an aerated stirred reactor. Therefore, this method allows an understanding of the kinetic stability of a biocatalyst at an early stage of process development, under industrially relevant operational conditions. The method was tested by investigating the kinetic stability of $\mathrm{NAD}(\mathrm{P}) \mathrm{H}$ oxidase at gas-liquid interfaces and it was found that the deactivation rate 
of this enzyme increased with an increase in gas-liquid interfacial area. It was also observed that for large gas-liquid interfacial areas, the power input per volume was the dominating cause of deactivation. The phenomena of enzyme deactivation at gas-liquid interfaces is still not fully understood thus, it is necessary to isolate the possible operational conditions that cause protein deactivation. Finally, a correlation between NOX half-life and specific gas-liquid interfacial area was successfully obtained under the experimental conditions. This correlation demonstrates that the approach developed here can be applied further to predict kinetic stability of enzymes in the presence of industrially relevant gasliquid interfacial areas. Additionally, the knowledge gained can be used to guide protein engineers to improve the biocatalyst.

\section{Acknowledgements}

The research for this work has received funding from the European Union (EU) project ROBOX (grant agreement $\mathrm{n}^{\circ}$ 635734) under EU's Horizon 2020 Programme Research and Innovation actions H2020-LEIT BIO-2014-1. Any statements herein reflect only the author's views. The European Union is not liable for any use that may be made of the information contained herein. The authors acknowledge Dr Christian Bach for his assistance in the development of the optical method and the Matlab code for the bubble size distributions analysis.

\section{Figures}
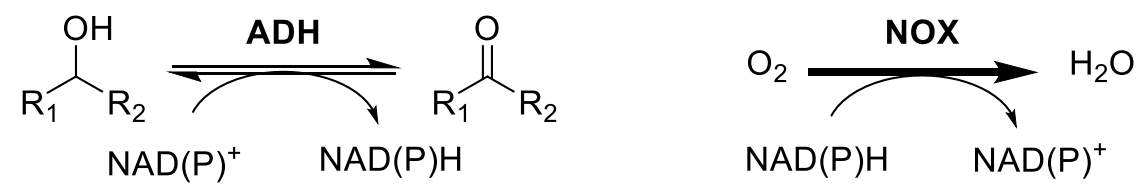

Figure 1. Oxidation of primary or secondary alcohols to ketones or aldehydes catalysed by an ADH. NOX system for nicotinamide cofactor regeneration. 

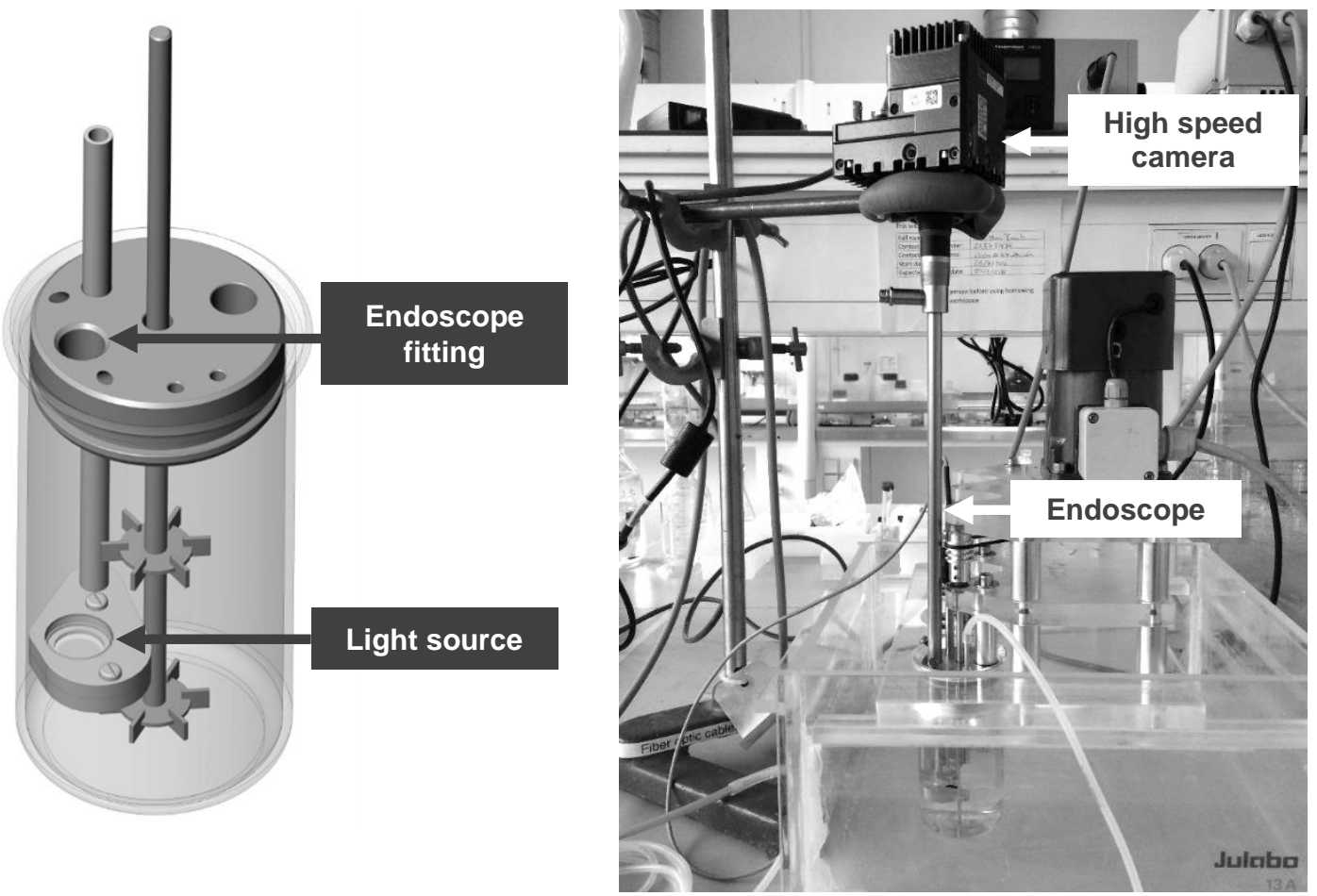

Figure 2. Setup of the bioreactor with an optical probe incorporated. (Left) Scheme of the reactor showing the two Rushton turbines and the light source. (Right) Picture of the complete setup highlighting the high speed camera and the endoscope. 


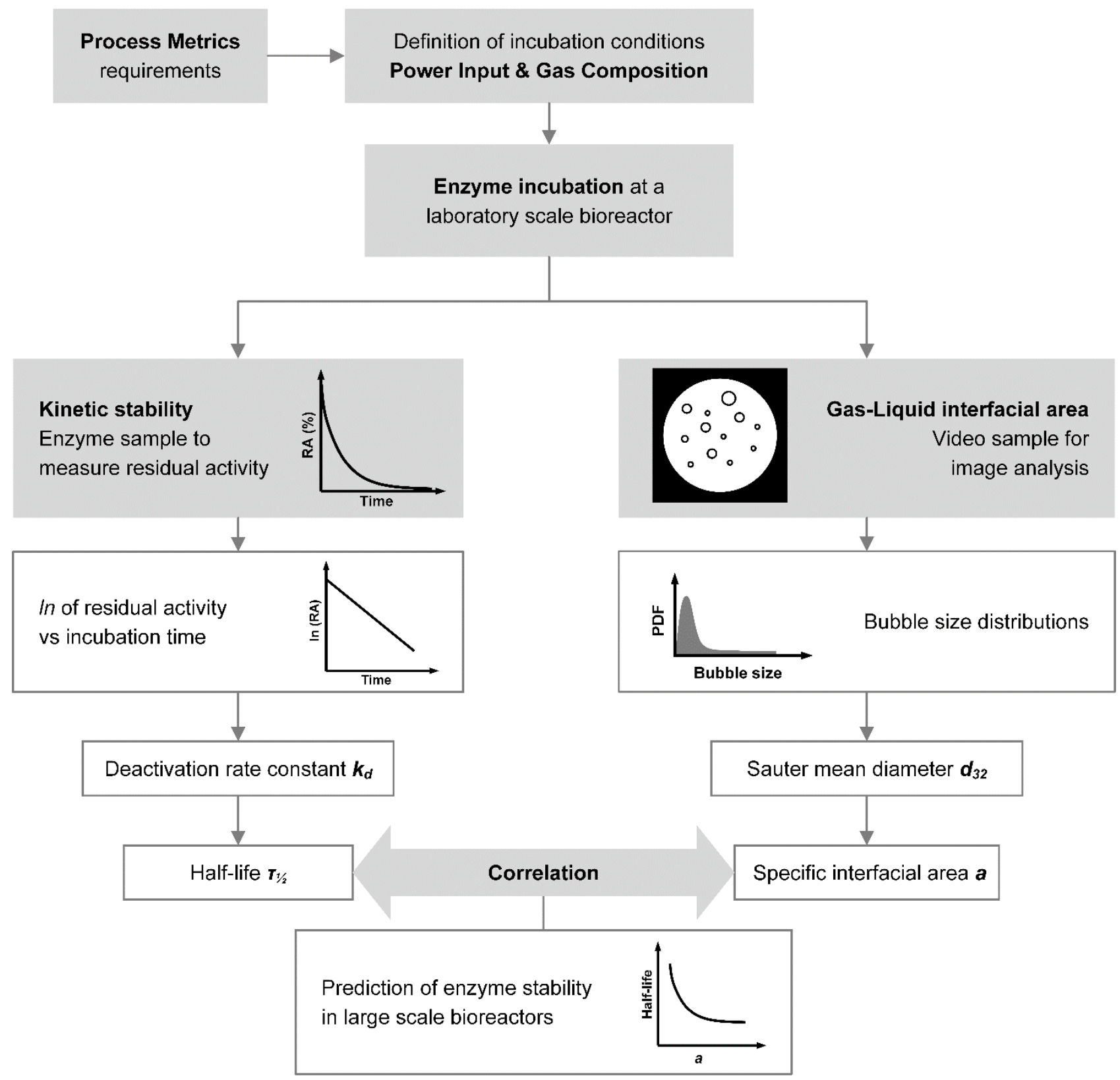

Figure 3. Data flow diagram of the methodology developed to determine enzyme stability at specific gas liquid interfacial areas achieved in large scale reactors. 


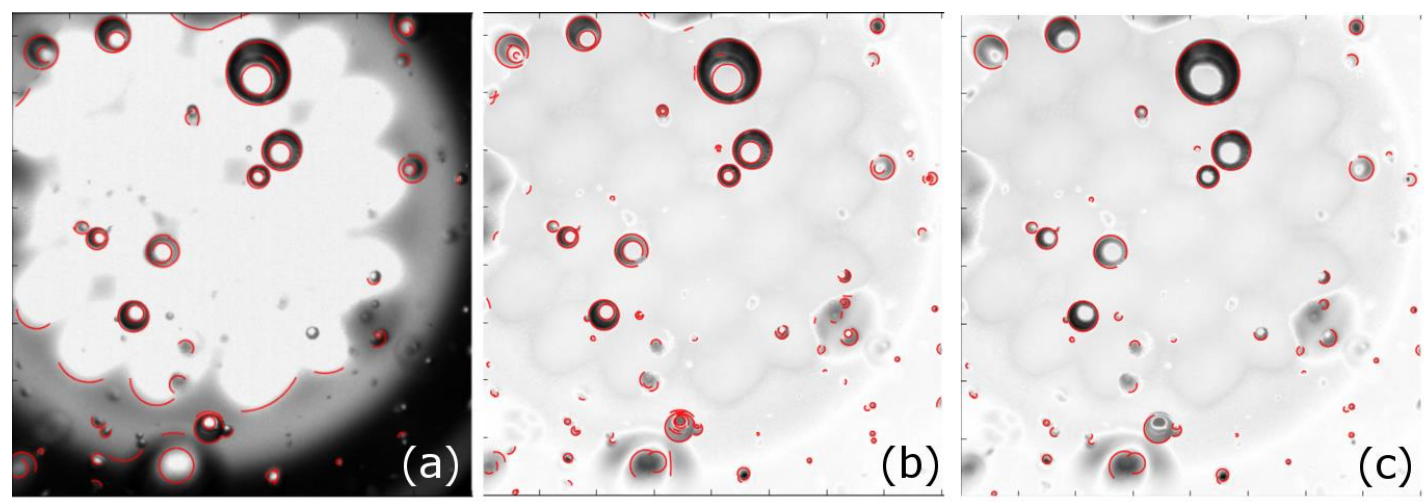

Figure 4. A typical frame from the setup under varying stages in the image processing with the red lines indicating detected objects characterized by having an elliptic shape. (a) is the original image, (b) is the image after upscaling to detect small bubbles, and background removal, (c) shows the result of eliminating double detection.

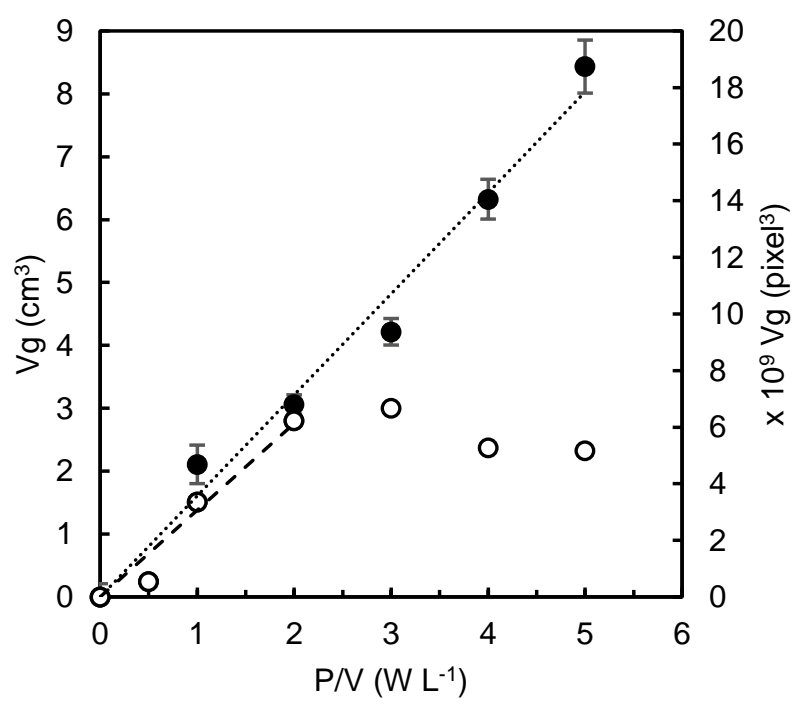

Figure 5. Volume of gas in the reactor $\left(\mathrm{V}_{\text {gas }}\right)$ determined using two methods: $(\bullet)$ estimation from experimentally measured gas holdups in $\mathrm{cm}^{3}$ and $(0)$ calculation using image analysis based on cubic pixels. 

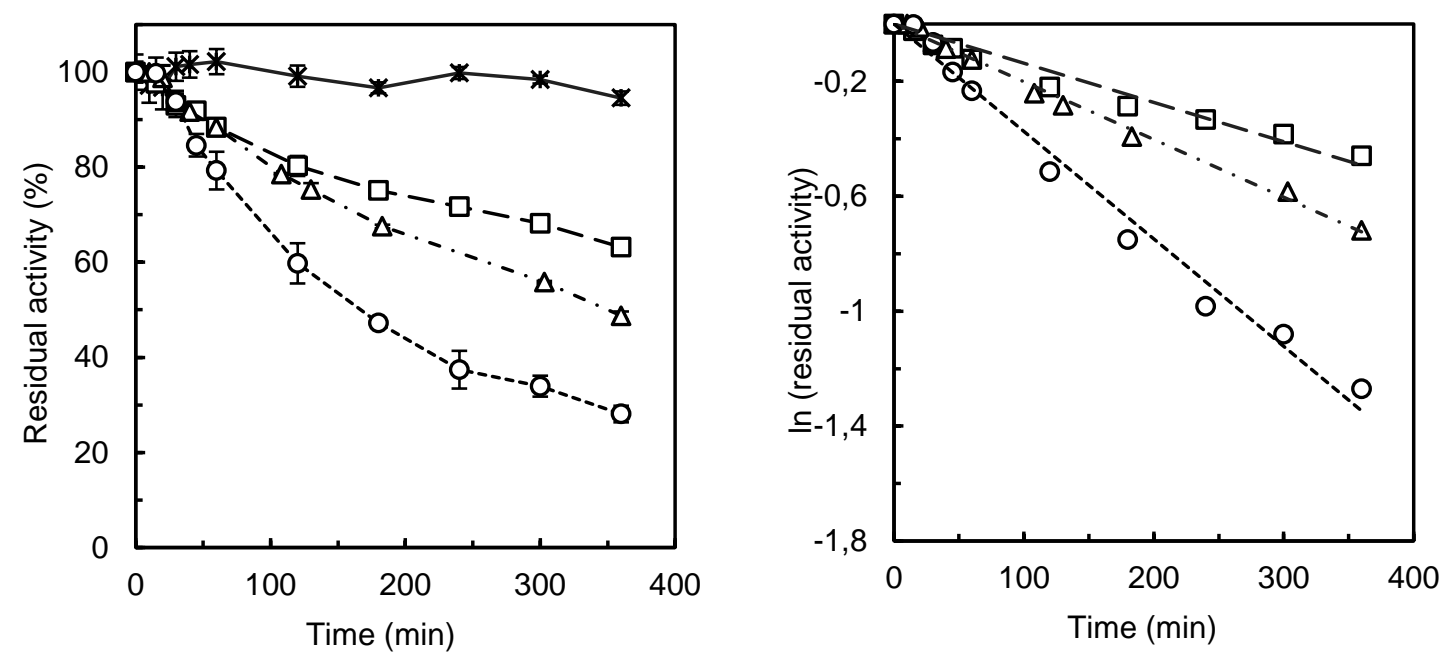

Figure 6. (Left) Residual activity of NOX at different P/V conditions: (×) Quiescent solution, $(\square) 0.5 \mathrm{WL}^{-1},(\triangle) 1 \mathrm{WL}^{-1}$, (O) $2 \mathrm{WL}^{-}$ 1 . Activity was measured in triplicate. Error bars reflect standard deviation and may not be visible since it was always below $\pm 5 \%$. (Right) First-order kinetic deactivation curve from the data presented in the left graph.
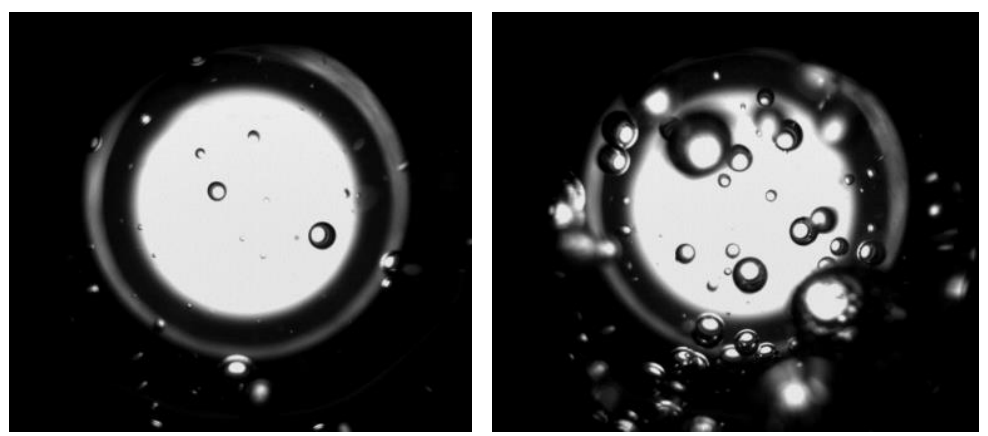

Figure 7. Regime of the bubbles inside the reactor at an aeration rate of $1 \mathrm{vvm}$ and different power inputs. $1 \mathrm{WL}^{-1}$ (left) and $2 \mathrm{WL}^{-1}$ (right). 

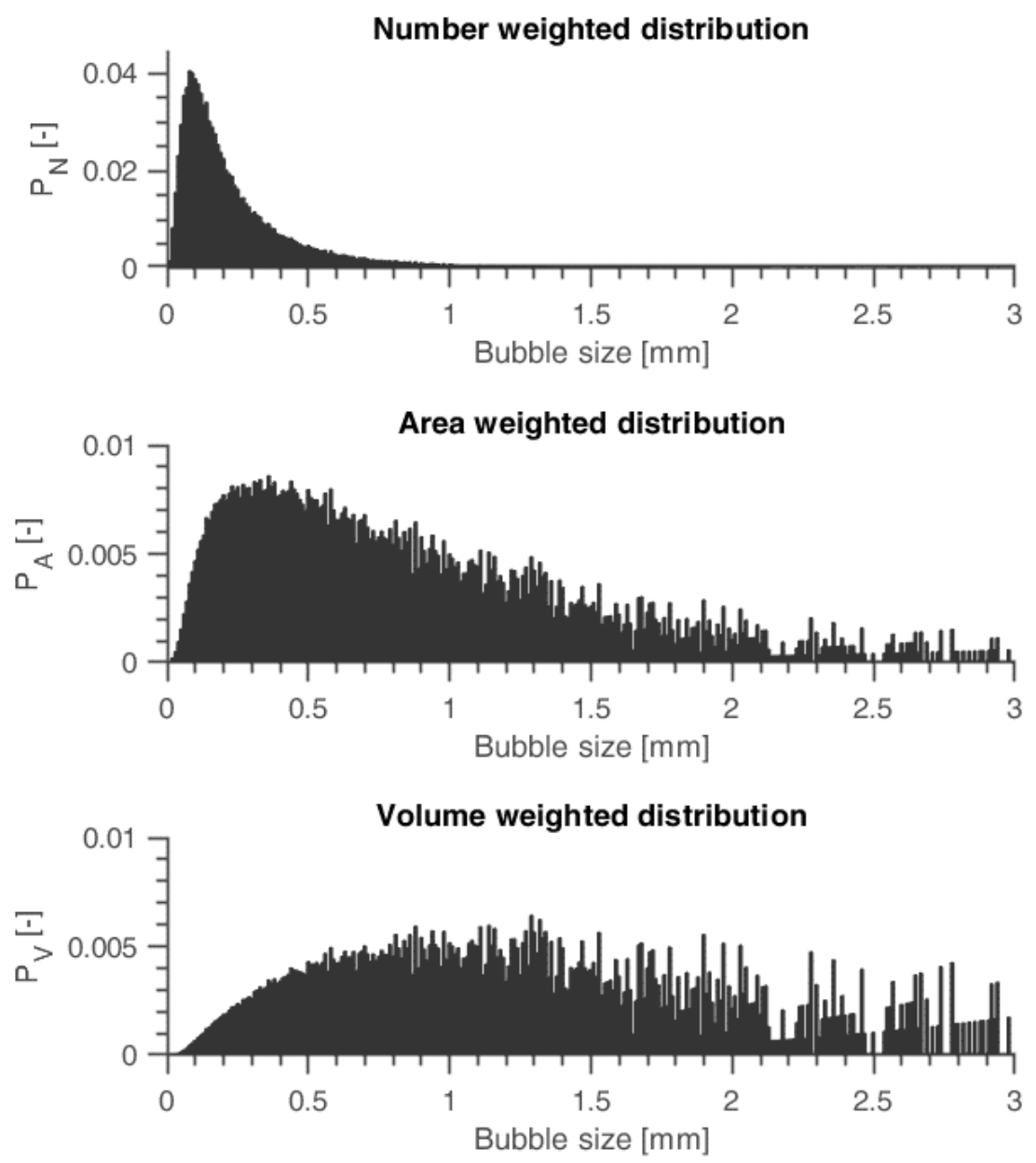

Figure 8. Bubbles' size distributions at a power input of $2 \mathrm{WL}^{-1}$ and a gas flow rate of $1 \mathrm{vvm}$. The distributions were calculated based on number, area and volume probability density functions of the bubbles' diameter $\left(P_{N}, P_{A}\right.$, and $\left.P_{V}\right)$. Distributions were discretized in 60 bins. 


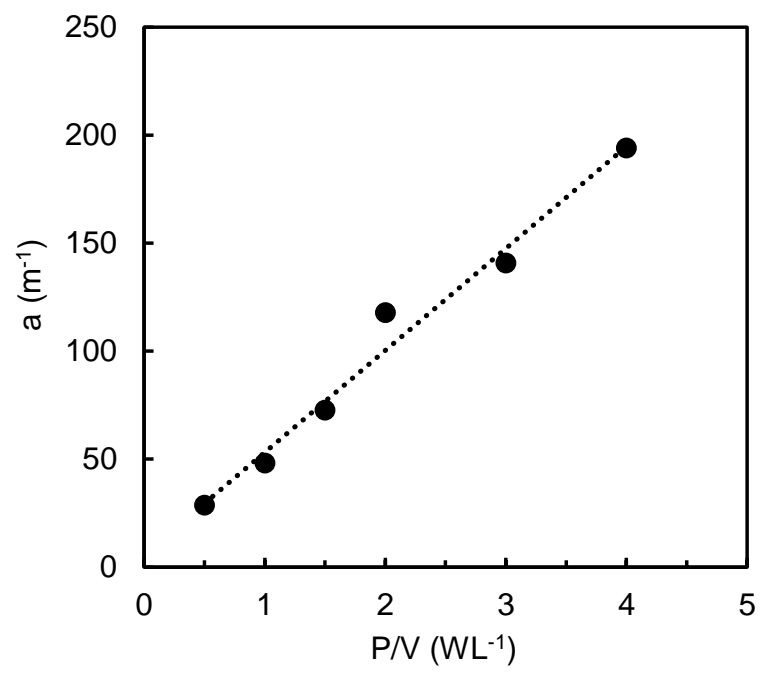

Figure 9. Correlation of specific interfacial area $(a)$ and power input per volume to the reactor $(\mathrm{P} / \mathrm{V})$. The line represents a linear regression with a $\mathrm{R}^{2}$ of $98 \%(\mathrm{P} / \mathrm{V}=47 \cdot \mathrm{P} / \mathrm{V}+5.9)$.

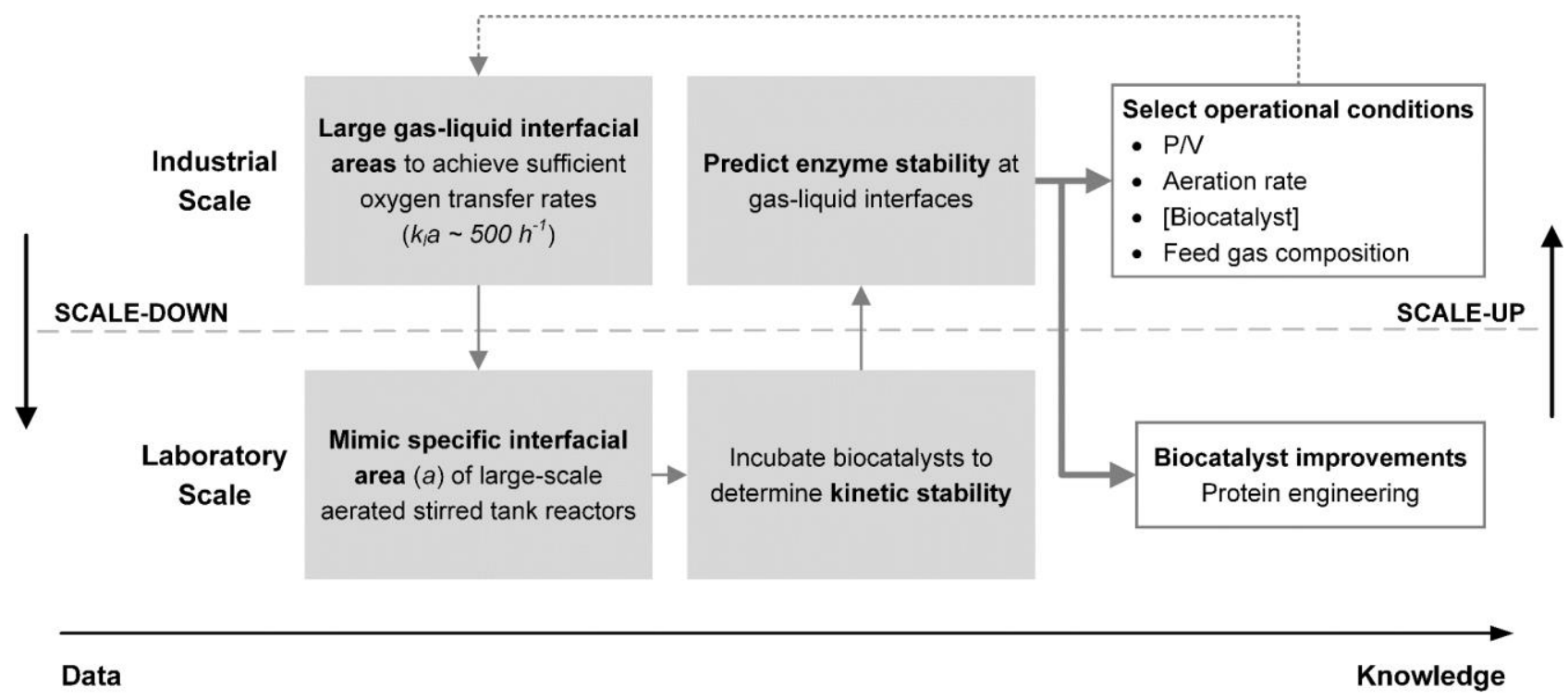

Figure 10. Scale-down approach to determine enzyme stability at gas-liquid interfacial areas similar to large scale bioreactors. 


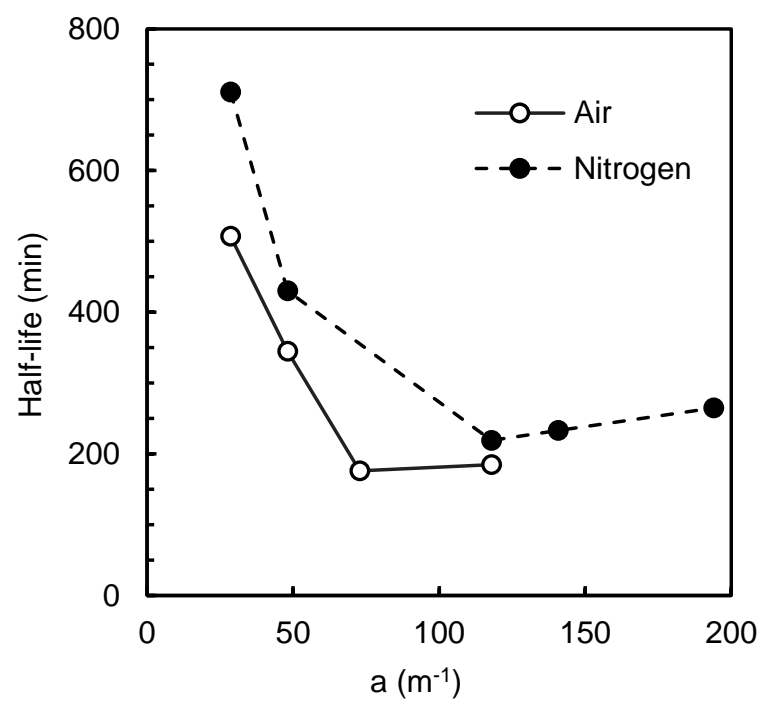

Figure 11. Half-life of NOX as function of different specific interfacial area conditions at a gas flow rate of $1 \mathrm{vvm}$.

\section{Tables}

Table 1. Deactivation rate constants $\left(k_{d}\right)$ and half-life of NOX at different power inputs per volume for non-aerated and aerated systems. Air $\left(21 \%\right.$ oxygen) and nitrogen were sparged at $1 \mathrm{vvm}$. The relative errors of the deactivation constants for power inputs up to $2 \mathrm{WL}^{-}$ ${ }^{1}$ were below $\pm 3 \%$ and for power inputs higher than $3 \mathrm{WL}^{-1}$ were below $\pm 8 \%$.

\begin{tabular}{ccccccc}
\hline \multirow{2}{*}{$\begin{array}{c}\text { Power input } \\
\left(\mathbf{W L}^{-1}\right)\end{array}$} & \multicolumn{2}{c}{ Air } & \multicolumn{2}{c}{ Nitrogen } & \multicolumn{2}{c}{ No Aeration } \\
\cline { 2 - 6 } & $k_{d}\left(\mathrm{~min}^{-1}\right)$ & Half-life $(\mathrm{min})$ & $k_{d}\left(\mathrm{~min}^{-1}\right)$ & Half-life $(\mathrm{min})$ & $k_{d}\left(\mathrm{~min}^{-1}\right)$ & Half-life (min) \\
\hline 0.5 & $1.4 \times 10^{-3}$ & 507 & $7.8 \times 10^{-4}$ & 711 & $9.1 \times 10^{-4}$ & 760 \\
1 & $2.0 \times 10^{-3}$ & 345 & $1.6 \times 10^{-3}$ & 430 & $1.6 \times 10^{-3}$ & 431 \\
1.5 & $4.0 \times 10^{-3}$ & 176 & - & - & $2.9 \times 10^{-3}$ & 235 \\
2 & $3.7 \times 10^{-3}$ & 185 & $3.2 \times 10^{-3}$ & 219 & & - \\
3 & - & - & $3.0 \times 10^{-3}$ & 233 & & - \\
4 & - & - & $2.6 \times 10^{-3}$ & 265 & & - \\
\hline
\end{tabular}

\section{References}

1. Martínez AT, Ruiz-Dueñas FJ, Camarero S, et al. Oxidoreductases on their way to industrial biotransformations. Biotechnol Adv. 2017;35:815-831.

2. Dong JJ, Fernández-Fueyo E, Hollmann F, et al. Biocatalytic Oxidation Reactions: A Chemist's Perspective. Angew Chemie - Int Ed. 2018;57(30):9238-9261. 
3. Hollmann F, Arends IWCE, Buehler K, Schallmey A, Bühler B. Enzyme-mediated oxidations for the chemist. Green Chem. 2011;13(2):226-265.

4. Turner NJ. Enantioselective Oxidation of C-O and C-N bonds using oxidases. Chem Rev. 2011;111(7):40734087.

5. Xu F. Applications of oxidoreductases: recent progress. Ind Biotechnol Spring. 2005;1:38.

6. Wang Y, Lan D, Durrani R, Hollmann F. Peroxygenases en route to becoming dream catalysts. What are the opportunities and challenges? Curr Opin Chem Biol. 2017;37:1-9.

7. Dumeignil F, Guehl M, Gimbernat A, et al. From sequential chemoenzymatic synthesis to integrated hybrid catalysis: Taking the best of both worlds to open up the scope of possibilities for a sustainable future. Catal Sci Technol. 2018.

8. Truppo MD. 7.4 Cofactor Recycling for Enzyme Catalyzed Processes. In: Comprehensive Chirality. Vol 7. ; 2012:46-70.

9. Liu J, Wu S, Li Z. Recent advances in enzymatic oxidation of alcohols. Curr Opin Chem Biol. 2018;43:77-86.

10. Brummund J, Sonke T, Müller M. Process Development for Biocatalytic Oxidations Applying Alcohol Dehydrogenases. Org Process Res Dev. 2015;19(11):1590-1595.

11. Hummel W, Gröger H. Strategies for regeneration of nicotinamide coenzymes emphasizing self-sufficient closedloop recycling systems. J Biotechnol. 2014;191:22-31.

12. Ferrandi EE, Monti D, Riva S. New Trends in the In Situ Enzymatic Recycling of NAD(P)(H) Cofactors. In: Cascade Biocatalysis: Integrating Stereoselective and Environmentally Friendly Reactions. ; 2014:23-42.

13. Kroutil W, Mang H, Edegger K, Faber K. Biocatalytic oxidation of primary and secondary alcohols. Adv Synth Catal. 2004;346(2-3):125-142. 
14. Rehn G, Pedersen AT, Woodley JM. Application of NAD(P)H oxidase for cofactor regeneration in dehydrogenase catalyzed oxidations. J Mol Catal B Enzym. 2016;134:331-339.

15. Burton SG. Oxidizing enzymes as biocatalysts. Trends Biotechnol. 2003;21(12):543-549.

16. Abu R, Woodley JM. Application of Enzyme Coupling Reactions to Shift Thermodynamically Limited Biocatalytic Reactions. ChemCatChem. 2015;7(19):3094-3105.

17. Tufvesson P, Lima-Ramos J, Nordblad M, Woodley JM. Guidelines and cost analysis for catalyst production in biocatalytic processes. Org Process Res Dev. 2011;15(1):266-274.

18. Woodley JM. Bioprocess intensification for the effective production of chemical products. Comput Chem Eng. 2017;105:297-307.

19. Sheldon RA, Woodley JM. Role of Biocatalysis in Sustainable Chemistry. Chem Rev. 2018;118(2):801-838.

20. Law HEM, Baldwin CVF, Chen BH, Woodley JM. Process limitations in a whole-cell catalysed oxidation: Sensitivity analysis. Chem Eng Sci. 2006;61(20):6646-6652.

21. Mohanty M, Ghadge RS, Patil NS, Sawant SB, Joshi JB, Deshpande A V. Deactivation of lipase at gas-liquid interface in stirred vessel. Chem Eng Sci. 2001;56(11):3401-3408.

22. Bommarius AS, Karau A. Deactivation of Formate Dehydrogenase (FDH) in solution and at gas-liquid interfaces. Biotechnol Prog. 2005;21(6):1663-1672.

23. Perriman AW, Henderson MJ, Holt SA, White JW. Effect of the air-water interface on the stability of betalactoglobulin. J Phys Chem B. 2007;111(48):13527-13537.

24. Van Hecke W, Haltrich D, Frahm B, et al. A biocatalytic cascade reaction sensitive to the gas-liquid interface: Modeling and upscaling in a dynamic membrane aeration reactor. J Mol Catal B Enzym. 2011;68(2):154-161.

25. Bhagia S, Dhir R, Kumar R, Wyman CE. Deactivation of Cellulase at the Air-Liquid Interface Is the Main Cause 
of Incomplete Cellulose Conversion at Low Enzyme Loadings. Sci Rep. 2018;8(1):1350.

26. Caussette M, Gaunand A, Planche H, Colombie S, Monsan P, Lindet B. Lysozyme inactivation by inert gas bubbling: kinetics in a bubble column reactor. Enzyme Microb Technol. 1999;24:412-418.

27. Junker B, Maciejak W, Darnell B, Lester M, Pollack M. Feasibility of an in situ measurement device for bubble size and distribution. Bioprocess Biosyst Eng. 2007;30(5):313-326.

28. Raimundo PM, Cartellier A, Beneventi D, Forret A, Augier F. A new technique for in-situ measurements of bubble characteristics in bubble columns operated in the heterogeneous regime. Chem Eng Sci. 2016;155:504523.

29. Petschacher B, Staunig N, Müller M, et al. Cofactor Specificity Engineering of Streptococcus mutans NADH Oxidase 2 for $\operatorname{NAD}(\mathrm{P})(+)$ Regeneration in Biocatalytic Oxidations. Comput Struct Biotechnol J. 2014;9:e201402005.

30. Paul EL, Atiemo-obeng VA, Kresta SM. Handbook of Industrial Mixing.; 2004.

31. Stocks SM. Industrial Enzyme Production for the Food and Beverage Industries: Process Scale up and Scale Down. Woodhead Publishing Limited; 2013.

32. Pătrăucean V, Gurdjos P, Von Gioi RG. A parameterless line segment and elliptical arc detector with enhanced ellipse fitting. In: Lecture Notes in Computer Science (Including Subseries Lecture Notes in Artificial Intelligence and Lecture Notes in Bioinformatics). Vol 7573 LNCS. ; 2012:572-585.

33. Lowe DG. Perceptual organization and visual recognition. Comput Vision, Graph Image Process. 1985;31(31):394.

34. Grompone von Gioi R, Jakubowicz J, Morel J-M, Randall G. LSD: a Line Segment Detector. Image Process Line. 2012. 
35. Whitman WG. The two-film theory of gas absortion. J Heat Mass Transf. 1962;5(1916):429-433.

36. Garcia-Ochoa F, Gomez E. Theoretical prediction of gas-liquid mass transfer coefficient, specific area and holdup in sparged stirred tanks. Chem Eng Sci. 2004;59(12):2489-2501.

37. Bach C, Yang J, Larsson H, et al. Evaluation of mixing and mass transfer in a stirred pilot scale bioreactor utilizing CFD. Chem Eng Sci. 2017;171:19-26.

38. Sadana A. Enzyme Deactivation. Biotechnol Adv. 1988;6(3):349-446.

39. Laakkonen M, Moilanen P, Miettinen T, et al. Local bubble size distributions in agitated vessel comparison of three experimental techniques. Chem Eng Res Des. 2005;83(1 A):50-58.

40. Jiang R, Riebel BR, Bommarius AS. Comparison of alkyl hydroperoxide reductase (AhpR) and water-forming NADH oxidase from Lactococcus lactis ATCC 19435. Adv Synth Catal. 2005;347(7-8):1139-1146.

41. Park JT, Hirano JI, Thangavel V, Riebel BR, Bommarius AS. NAD(P)H oxidase v from Lactobacillus plantarum (NoxV) displays enhanced operational stability even in absence of reducing agents. J Mol Catal B Enzym. 2011;71(3-4):159-165.

42. Dias Gomes M, Bommarius BR, Anderson SR, Feske BD, Woodley JM, Bommarius AS. Bubble Column Enables Higher Reaction Rate for Deracemization of ( R , S ) -1-Phenylethanol with Coupled Alcohol Dehydrogenase / NADH Oxidase System. Adv Synth Catal. 2019;361:2574-2581. 